\title{
Micro-to-macro fluidic interconnectors with an integrated polymer sealant
}

\author{
Jr-Hung Tsai ${ }^{1,2,3}$ and Liwei Lin ${ }^{2}$ \\ ${ }^{1}$ Mechanical Engineering, University of Michigan, Ann Arbor, MI 48109-2125, USA \\ ${ }^{2}$ Mechanical Engineering, 5126 Etcheverry Hall, University of California, Berkeley, CA \\ 94720-1740, USA \\ E-mail: jhtsai@argon.eecs.berkeley.edu
}

Received 26 February 2001, in final form 14 June 2001

Published 9 August 2001

Online at stacks.iop.org/JMM/11/577

\begin{abstract}
This paper presents the design, fabrication and testing results of inserting a polymer sealant (Mylar) into a micro-to-macro fluidic interconnection. Two processes, discrete and integrated Mylar sealant, have been developed by means of post-fabrication after the mircofluidic components such as micro-channels and micro-chambers are constructed. The integrated process utilizes microfabrication techniques such that batch processing is feasible and the sealant dimensions can be precisely controlled. The discrete process takes advantage of easy and simple processing and is compatible with most microfluidic systems without any extra wafer-level micromachining process. In both processes, macroscale capillary tubes with a diameter of $320 \mu \mathrm{m}$ have been successfully connected to microscale channels with the help of the Mylar. Both leakage and pull-out tests are conducted and successfully demonstrate the functionality of the interconnectors. The leakage test shows that no leakage is observed up to $190 \mathrm{kPa}$ and the pull-out test proves $100 \%$ survival rate under a pulling force of $2 \mathrm{~N}$.
\end{abstract}

(Some figures in this article are in colour only in the electronic version)

\section{Introduction}

Micro-to-macro fluidic interconnectors are indispensible elements in miniaturized fluidic systems such as micropumps $[1,2]$, microvalves [3], or even microchromatographic devices [4]. Previously, capillary tubes have been connected to microfluidic devices by means of fitting to precision-machined holes [1] or microfabricated openings [3-6]. Adhesives are generally used after the assembly process of these interconnectors to prevent leakage. In order to simplify the manufacture and assembly processes, various attempts have been reported to improve the micro-to-macro interconnection processing. For example, Wijngaart et al [2] developed a method by directly melting polyethylene capillary tubes on the interconnectors and then applying epoxy on the interconnection area. Yao et al [7] used silicone rubber as O-ring couplers for reusable interconnectors. Since silicone rubber must be introduced as part of the fabrication process,

3 Author to whom correspondence should be addessed. this approach may not be compatible with various microfluidic systems.

This paper presents two alternative approaches, integrated and discrete processes, by introducing a sealing polymer layer (Mylar) to assist in the micro-to-macro interconnection process.

\section{Fabrication processes}

A Mylar (DuPont Mylar 50M44) film is introduced in both processes. A microfluidic system consisting of a microchannel and two micro-to-macro fluidic ports is first constructed as shown in figure 1 . In the process, a DRIE (deep reactive ion etching) etcher is used to make $20 \mu \mathrm{m}$ deep microchannels in a silicon wafer as illustrated in figures $1(a)$ and $(b)$. Fluidic interconnectors are fabricated on a $500 \mu \mathrm{m}$-thick Pyrex glass wafer and a photoresist is used to define the position of the fluidic ports as shown in figure 1(c). A conventional mechanical drilling process is then used to open the circular ports of about $350 \mu \mathrm{m}$ in diameter through the $500 \mu \mathrm{m}$-thick 


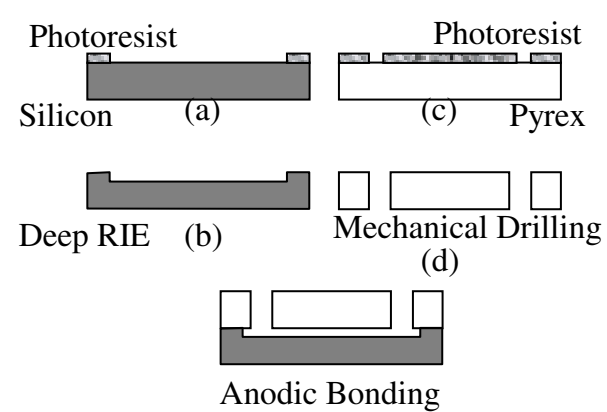

(e)

Figure 1. Fabrication process of the microchannel.

Pyrex glass wafer as shown in figure $1(d)$. The silicon and glass substrates are aligned and bonded together using an anodic bonding technique; figure 1(e) applies after these processes. Thereafter, discrete and integrated processes are conducted to introduce the polymer sealant, as shown in figure 2 .

In the discrete process, the Mylar film, $14 \mu \mathrm{m}$ thick, is manually cut into tiny square pieces $3000 \mu \mathrm{m}$ in width. The capillary tubes with an outer diameter of $320 \mu \mathrm{m}$ are used to penetrate the Mylar film as shown in figure 2(a.l). This assembly is put into the fluidic port on the glass substrate. The penetration depth of the assembly is adjusted manually such that the capillary tube does not touch the bottom of the microchannel. The polyvinylidene chloride (PVDC) coating on the Mylar film must face the glass substrate to function as the bonding adhesive. The whole system is then put on a hot plate at $180^{\circ} \mathrm{C}$ for $5 \mathrm{~min}$ to activate the PVDC bonding; the system is naturally cooled following the heating process. After these steps, figure 2(a.2) applies. A droplet of room-temperature curing, instant glue (SUR-LOK) of a viscosity of around $20 \mathrm{cps}$ (centipoise) is applied around the interconnection hole to enhance the holding force; figure 2(a.3) shows the completed process.

The integrated process starts from attaching the Mylar film to the wafer by a thermal bonding process. The Mylar film is cut into a 4 inch diameter circular shape to match the size of a 4 inch wafer and the surface, with PVDC coating, is placed in contact with the glass/silicon microchannel assembly. A second glass wafer is then placed on top of the Mylar film, facing the side without PVDC coating, to assist the bonding process by using the flatness and weight of the second glass wafer. The bonding processes takes place when the wafer is heated to and maintained at $160{ }^{\circ} \mathrm{C}$ for $15 \mathrm{~min}$ to activate the PVDC coating. The heating temperature is lower and time is longer than in the discrete process to minimize trapped air in this wafer-level bonding process. However, even with these tuned bonding conditions, trapped air bumps in the range of around hundreds of micrometres in diameter are scattered in the large flat surface areas. After the Mylar film is bonded onto the wafer, a $9 \mu \mathrm{m}$-thick photoresist (Shipley 1075) is spun on, patterned, and hard baked at $120^{\circ} \mathrm{C}$ for $30 \mathrm{~min}$ as an etching mask. Figure $2(b . l)$ applies after these steps. A $300 \mathrm{~W}$ oxygen plasma is then applied to pattern the Mylar film and to simultaneously remove the masking photoresist. The etching rates of the photoresist and Mylar in the oxygen plasma are characterized by measuring the groove depths of two test samples every 10 min as shown in figure 3 . It is found that the etching rates of the Mylar film and the hard-baked photoresist are similar at about $0.35 \mu \mathrm{m} \mathrm{min}^{-1}$. Therefore, after etching through the opening ports on the $14 \mu \mathrm{m}$-thick Mylar film, the remaining Mylar film that was masked by the $9 \mu \mathrm{m}$-thick photoresist has a thickness of around $9 \mu \mathrm{m}$. The remaining Mylar is designed as the integrated polymer sealant to assist in the micro-to-macro interconnection. Capillary tubes are then fitted through the polymer sealant, and the tube inserting process can be automated in parallel as illustrated in figure 2(b.3). Finally, the same glue as used in the discrete process is applied to enhance the bonding strength and to complete the interconnection fabrication process, as shown in figure 2(b.4)

The insertion direction of the capillary tube has a direct implication on the sealing of the interconnector, as illustrated in figure 4 . In the discrete process, the capillary tube pierces through the Mylar film in an upward manner. During the Mylar/glass bonding process, molten Mylar surrounding the capillary tube could seal the gap between the capillary tube and

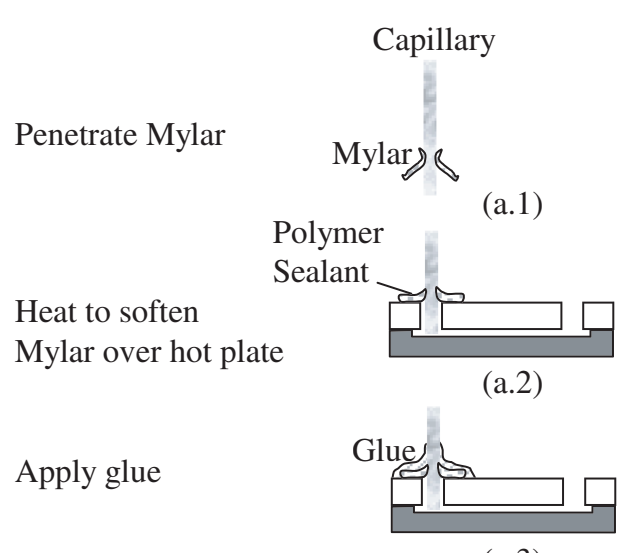

(a.3)
Heat to attach Mylar on the assembly and spin on PR

Pattern Mylar by $\mathrm{O}_{2}$ plasma

Put capillary through holes

Apply glue
Photoresist

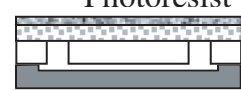

(b.1)

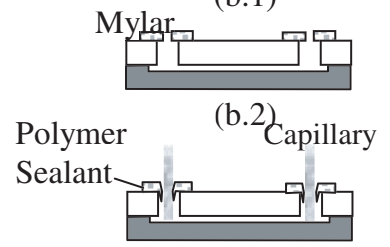

(b.3)

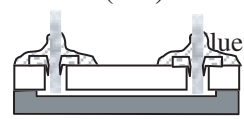

(b.4)

(a)

(b)

Figure 2. Process flows as a demonstration of the concept: $(a)$ discrete processing and $(b)$ integrated processing. 


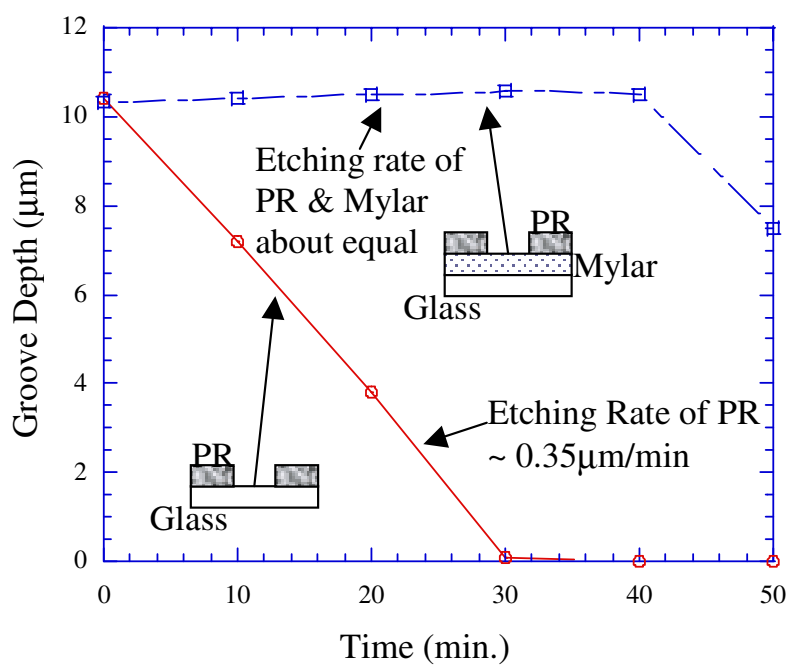

Figure 3. The depths of two types of grooves are measured with respect to the etching time in the $\mathrm{O}_{2}$ plasma etching process at $300 \mathrm{~W}$. It is found that the etching rates of the Shipley 1075 photoresist and the Mylar are about the same at $0.35 \mu \mathrm{m} \mathrm{min}^{-1}$.

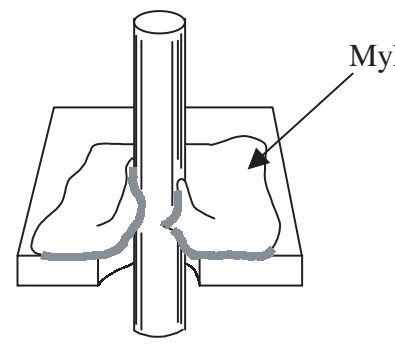

(a)

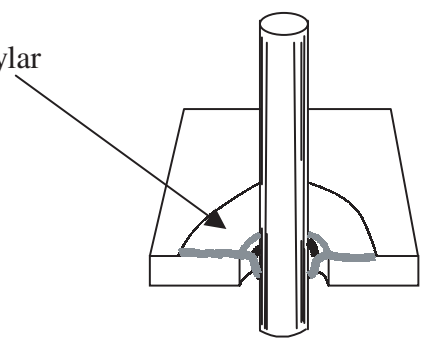

(b)
Figure 4. Schematic drawing of the sealing mechanism in $(a)$ the discrete and $(b)$ the integrated processes.

the microfluidic system. In the integrated process, the capillary tube is squeezed downwards through the fluidic ports as shown in figure $4(b)$. Therefore, if properly designed, the Mylar film works as an O-ring-type sealant between the tube and the fluidic port to seal the gap. In the prototype demonstration, the diameter of the capillary tube, the Mylar opening, and the fluidic ports are 320,300 , and $350 \mu \mathrm{m}$, respectively. This allows about a $20 \mu \mathrm{m}$-wide Mylar strip to be squeezed inside the fluidic port as sealant.

\section{Interconnector characterization}

\subsection{Visual inspection}

Scanning electron microscopy (SEM) micrographs of the interconnection areas were taken before the glue was applied for both the discrete and integrated processes, as shown in figures 5(a) and $(b)$, respectively. It is observed that the Mylar layer melts and covers the gap well in the discrete process, but leakage may exist and glue should be applied afterwards to complete the sealing. On the other hand, the Mylar sealant is squeezed downwards, inside the fluidic port, in the integrated process as shown in figure $5(b)$ and an extra glue cover is necessary to achieve a complete seal. Due to the oxygen

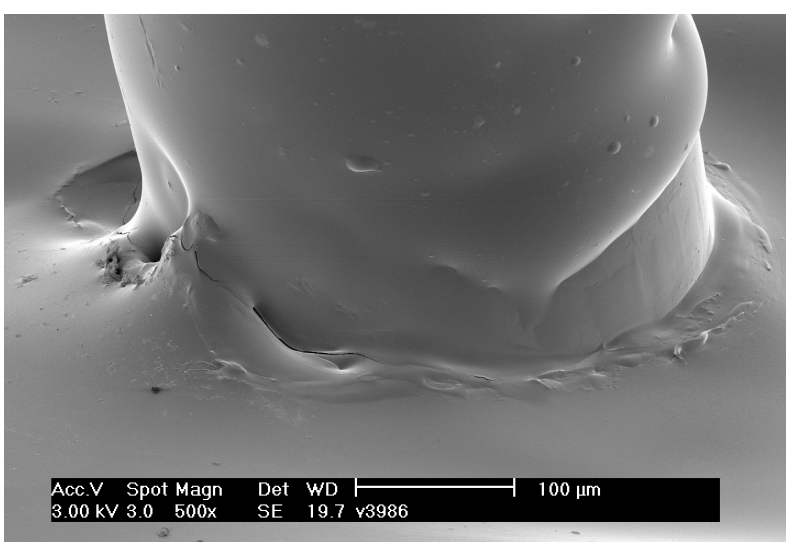

(a)

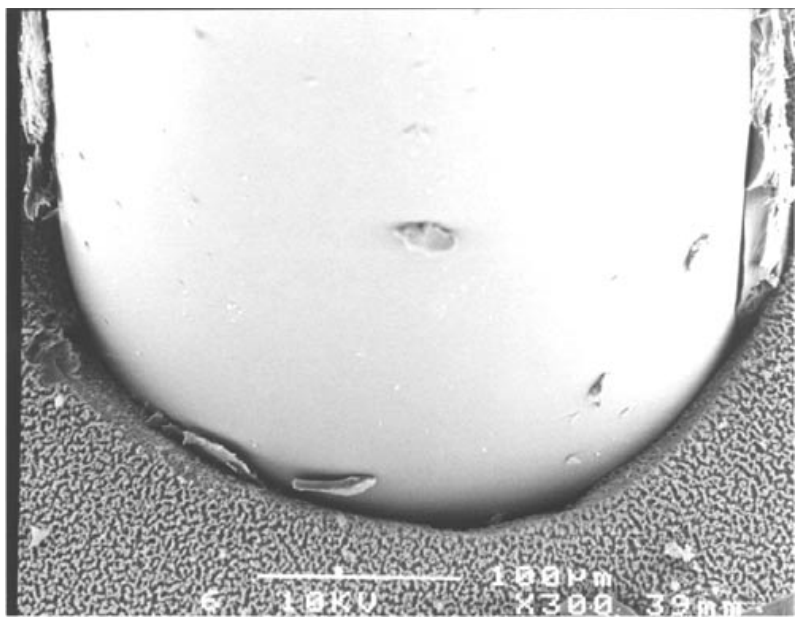

(b)

Figure 5. Close-up view SEM micrograph showing the micro-to-macro interconnectors: $(a)$ interconnection made by the discrete process and $(b)$ interconnection made by the integrated process.

plasma, the surface of the Mylar is rough, as observed. Both the discrete and integrated processes provide a first-level seal before a layer of glue is applied to improve the sealing and mechanical strength. The primary concern in most microto-macro fluidic interconnections is that the glue may seep through the gaps and block the capillary tubes; the polymer sealant provides a first-level seal to prevent the seeping of glue.

Figure 6 shows a SEM micrograph taken from the back side of the interconnector after glue is applied. Irregular cuts on the capillary tube can be seen as compared with the relatively smooth surface on the glass substrate. The outer diameter of the tube is $320 \mu \mathrm{m}$ and the gap between the capillary tube and the glass substrate is estimated as $15 \mu \mathrm{m}$. As can be observed by the micrograph, no glue can be identified, as the glue is blocked by the Mylar sealant. Because glue provides the major mechanical holding force on the capillary tubes, it must contact and adhere with both the capillary tube and the Pyrex glass wafer. Therefore, the glue must be dispensed to cover an area larger than the boundary of the Mylar film. Figures 7(a) and $(b)$ show the results of micro-to-macro interconnectors after the glue is applied. In the discrete process, a large area, larger than $3000 \mu \mathrm{m}$ in diameter, has to be used to cover the 


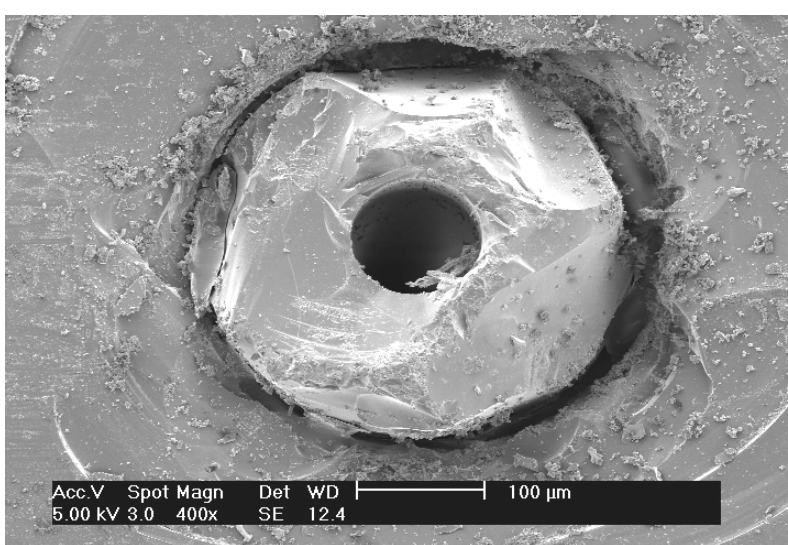

Figure 6. SEM micrograph of the back side of the interconnection by discrete processing. No blockage is observed at the opening of the capillary tube.

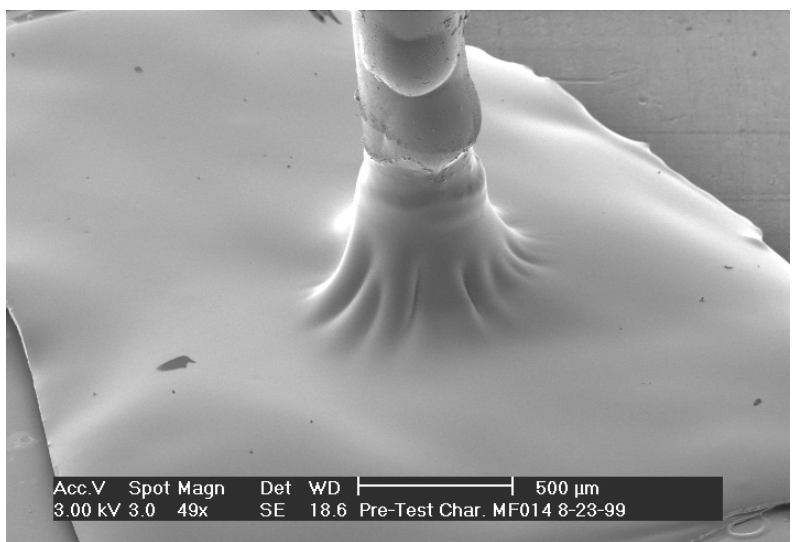

(a)

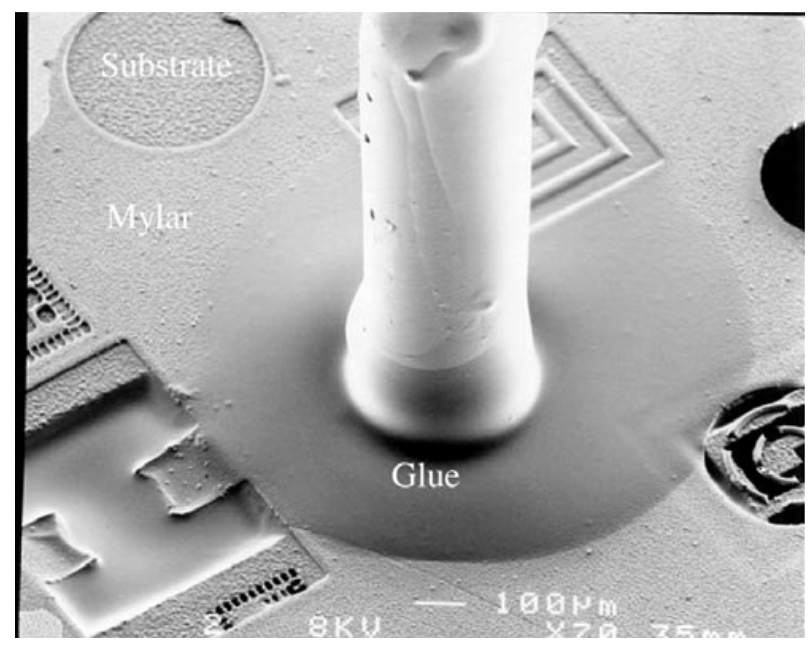

(b)

Figure 7. Interconnection areas after glue is applied: (a) interconnection made by the discrete process and (b) interconnection made by the integrated process.

Mylar piece. In contrast, the size of the Mylar film can be well controlled in the integrated process. For example, figure 7(b) shows glue of $1200 \mu \mathrm{m}$ diameter covering the $400 \mu \mathrm{m}$ outer diameter of the Mylar sealant.

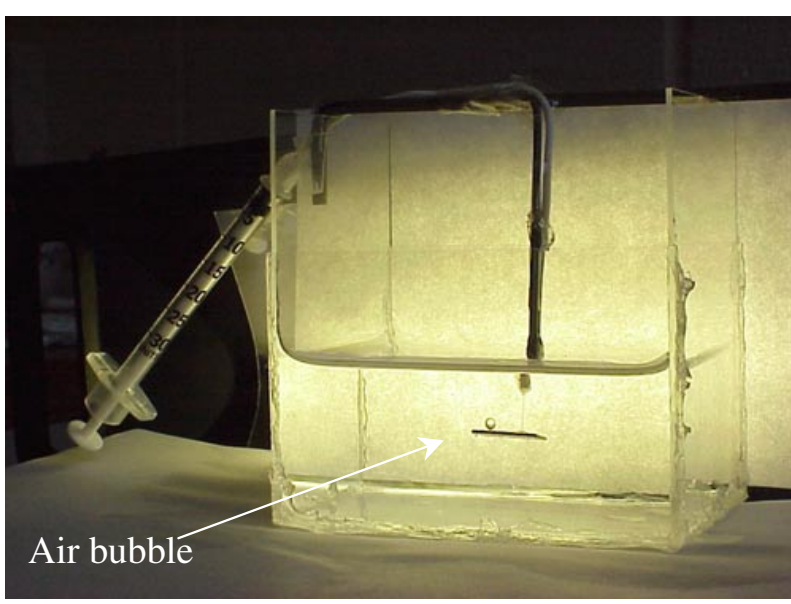

Figure 8. Photograph of the air injection test. Air is injected through the capillary tube into the microchannel. A bubble is seen at the fluidic outlet port.

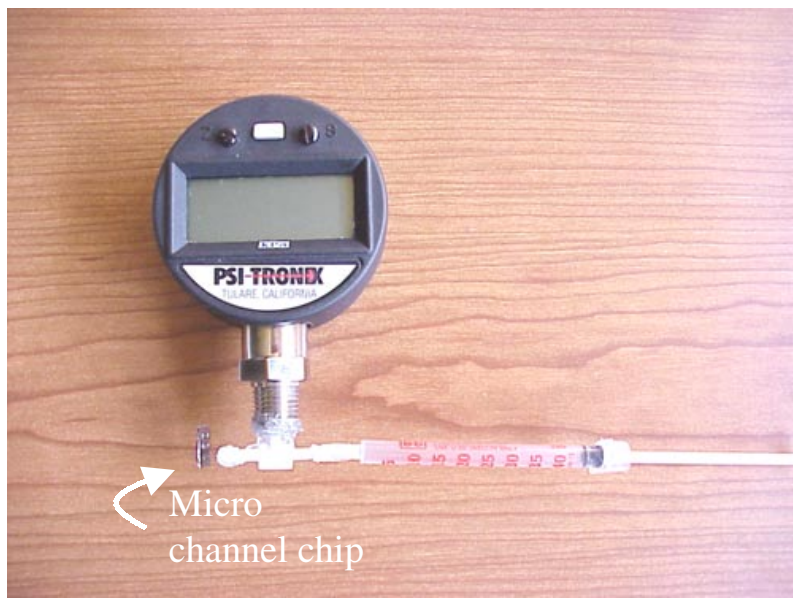

Figure 9. Photograph of the experimental set-up of the quantitative air pressure test.

\subsection{Leakage test}

Leakage tests are performed to characterize the seal of the micro-to-macro interconnectors. The preliminary leakage test is conducted by connecting a syringe via one interconnector to a microfluidic system that has a microchannel that is $800 \mu \mathrm{m}$ in length, $100 \mu \mathrm{m}$ in width, and $20 \mu \mathrm{m}$ in depth. The whole system, including the interconnectors, is immersed in water to detect air leakage by observing air bubbles when air is compressed in the microfluidic system by a syringe. Figure 8 shows the test set-up and the test result. It is found that air bubbles only appear at the other outlet of the microchannel and this qualitatively demonstrates that there is no leakage at the Mylar sealed interconnector.

The set-up of the second leakage experiment for quantitative measurement is shown in figure 9. The microinterconnector chip is coupled with a pressure transducer and a syringe by a three-way connector. The other outlet port of the microfluidic system is sealed by silicone. The pressure in the system is steadily increased by depressing the syringe cylinder, and both the volume and the pressure are recorded. As can be observed in figure 10, the pressure is proportional to the reciprocal of the enclosed volume of the syringe and the 


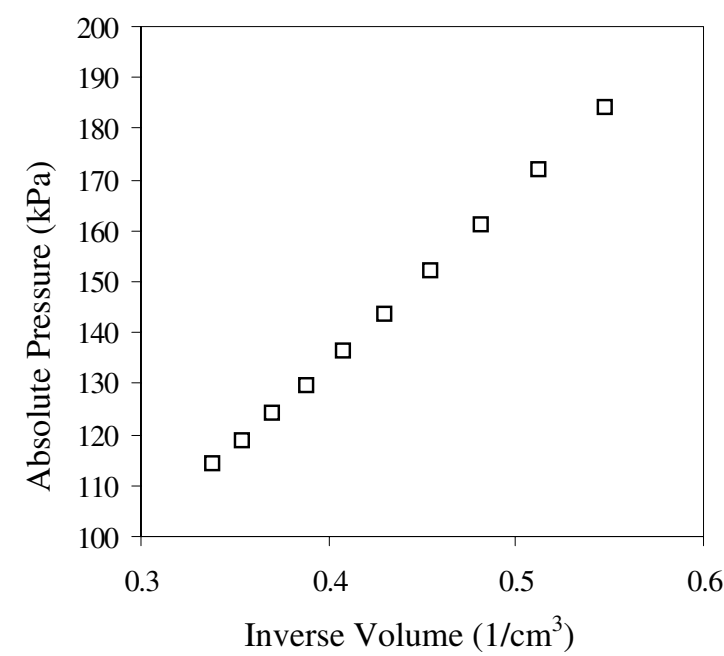

Figure 10. Pressure versus inverse volume for the interconnector test. A linear relationship between the pressure and the reciprocal of volume is found.

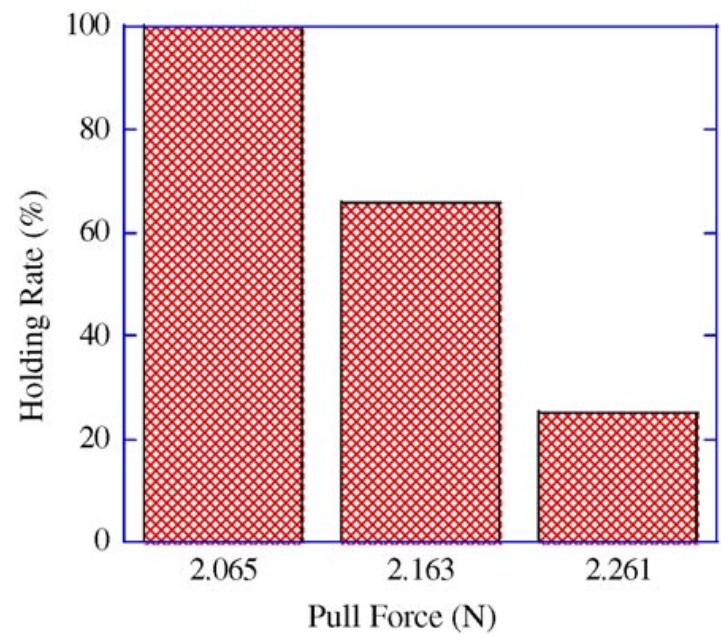

Figure 11. Pull-out test of sample assembled by the integrated process.

microfluidic system in the test pressure range can be predicted by the ideal gas law,

$$
P V=n R T
$$

where $P$ is the pressure, $V$ is the volume, $n$ is the mole number, $R$ is the universal gas constant, and $T$ is the temperature. The linear response between the pressure and the enclosed volume implies zero leakage up to the maximum test pressure, $190 \mathrm{kPa}$.

\subsection{Pull-out test}

The required force to separate the interconnector from the microsystem is characterized by a pull-out test. Weights are attached to the end of the capillary tube that is connected to the microchannel chip via the microinterconnector and a total of 12 samples are tested under different load conditions. The statistical testing results are shown in figure 11 and it is found that all tested samples survive under a constant pulling load of $2.065 \mathrm{~N}$. Four of the tested samples are broken when the load

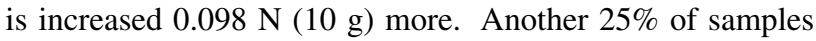
fail the test when the loading force reaches $2.261 \mathrm{~N}(230.7 \mathrm{~g})$.

\section{Conclusions}

Both discrete and integrated processes have been developed to apply polymer as a sealant in assisting micro-to-macro fluidic interconnections. These processes can be conducted after the completion of the microfluidic devices, with a maximum processing temperature of $180^{\circ} \mathrm{C}$ if Mylar is used as the polymer sealant. As such, they have potential applications in the area of micro-to-macro interconnectors. Both leakage and pull-out tests at the system level show the successful interconnections have been accomplished with the polymer sealant. Experimentally, up to $190 \mathrm{kPa}$ of pressure without leakage and up to $2.065 \mathrm{~N}$ of constant pulling force for at least 30 min without failure have been achieved. The developed micro-to-macro fluidic interconnection with polymer sealant provides an alternative approach to connect microfluidic devices with the external macroworld.

\section{Acknowledgments}

The authors would like to thank the microlab at the University of California at Berkeley for providing access to all the required microfabrication equipment and Dr Micheal Cohn for providing information on the Mylar film. This work is supported in part by a DARPA/MTO/MEMS grant (F3060298-2-0227).

\section{References}

[1] Olsson A, Enoksson P, Stemme G and Stemme E 1995 A valve-less planar pump in silicon Proc. Transducers '95 (Stockholm, Sweden, 1995) pp 291-4

[2] Wijngaart W, Andersson H, Enoksson P, Noren K and Stemme G 2000 The first self-priming and bi-directional valve-less diffuser micropump for both liquid and gas Proc. MEMS 2000 (Miyazaki, Japan, 2000) pp 674-9

[3] Shoji S, Schoot B V, Rooij N and Esashi M 1991 Smallest dead volume microvalves for integrated chemical analyzing systems Proc. Transducers '91 (Piscataway, NJ, 1991) pp 1052-5

[4] Hannoe S, Sugimoto I, Yanagisawa K and Kuwano H 1997 Enhanced chromatographic performance of silicon-micromachined capillary colume with clean structure and interactive plasma organic films Digest of Technical Papers, Transducers '97 (Chicago, IL, 1997) vol 1, pp 515-18

[5] Spiering V L, van der Moolen J N, Burger G J and van den Berg A 1997 Novel microstructures and technologies applied in chemical analysis techniques Digest of Technical Papers, Transducers '97 (Chicago, IL, 1997) vol 1, pp 511-14

[6] Jaeggi D, Gray B L, Mourlas N J, van Drieenhuizen B P, Williams K R, Maluf N I and Kovacs G T A 1998 Novel interconnection technologies for integrated microfluidic systems Proc. Solid-State Sensor and Actuator Workshop '98 (Hilton Head, MA, 1998) pp 112-15

[7] Yao T J, Lee S, Fang W L and Tai Y C 2000 Micromachined rubber O-ring micro-fluidic couplers Proc. MEMS 2000 (Miyazaki, Japan, 2000) pp 624-7 